\title{
Distribution-Free Tests for Two-Sample Location Problems Based on Subsamples
}

\author{
Deepa R. Acharya ${ }^{1}$ and Parameshwar V. Pandit ${ }^{2 *}$ \\ ${ }^{1}$ Department of Statistics, Govt. Science College, Bangalore -560001 \\ ${ }^{2}$ Department of Statistics, Bangalore University, Bangalore-560056 \\ Email: panditpv12@gmail.com
}

\begin{abstract}
Nonparametric tests for location problems have received much attention in the literature. Many nonparametric tests have been proposed for one, two and several samples location problems. In this paper a class of test statistics is proposed for two sample location problem when the underlying distributions of the samples are symmetric. The class of test statistics proposed is linear combination of U-statistics whose kernel is based on subsamples extrema. The members of the new class are shown to be asymptotically normal. The performance of the proposed class of tests is evaluated using Pitman Asymptotic Relative Efficiency. It is observed that the members of the proposed class of tests are better than the existing tests in the literature.
\end{abstract}

Keywords: Asymptotic relative efficiency, two-sample location problems, Nonparametric Tests, Symmetric distributions, U-statistics.

\section{Introduction}

Let $X_{1}, X_{2}, \ldots, X_{m}$ and $Y_{1}, Y_{2}, \ldots, Y_{n}$ be two independent random samples from two populations with continuous distribution functions $F(x)$ and $G(y)=F(x-\theta)$ respectively. Consider two sample location problem of testing $H_{0}: G(x)=F(x)$ for all $x$ against the alternative $H_{1}: G(x)=F(x-\theta)$, with $\theta \neq 0$ for some unknown continuous distribution function $\mathrm{F}$ and a real (shift) parameter $\theta,-\infty<\theta<\infty$. In the above testing problem, we may also consider one sided say, right sided alternative $H_{1}: \theta>0$. There are many nonparametric tests available in the literature for the above problem. Mann-Whitney's test [7] is a popular nonparametric procedure for this problem. Mood's median $(\mathrm{M})$ test [3] is effective in detecting shift in location in populations whose distributions are symmetric and heavy tailed. Gastwirth's H and L tests [2] are effective in detecting shifts in moderately heavy tailed distributions. The Normal Scores (NS) test [3] is effective in detecting a shift in the normal distribution. The RS test due to Hogg, Fisher and Randles [4] is effective in detecting shifts in distributions that are skewed. The SG test proposed by Shetty and Govindarajulu [10] based on subsample medians takes care of two suspected outliers at the extremes of both the samples. A generalization of Mathisen [8] is considered by Shetty and Bhat [11]. Their relative efficiency and suitability depends on the nature of the (unknown) underlying distribution F. Ahmad [1] proposed a generalization of Mann-Whitney test for this problem.

Section 2 contains the new proposed class of tests for two sample location problem. The distribution theory of the class of test statistics is presented in Section 3. The asymptotic relative efficiency comparisons are discussed in Section 4. In section 5 some remarks and conclusions are presented. 


\section{Proposed Class of Test Statistics}

Let $X_{1}, X_{2}, \ldots, X_{m}$ and $Y_{1}, Y_{2}, \ldots, Y_{n}$ be two independent random samples from two populations with continuous distribution functions $F(x)$ and $G(y)=F(x-\theta)$ respectively. Consider two sample location problem of testing $H_{o}: F(x)=G(x)$ for all $x$ against the alternative $H_{1}: G(x)=F(x-\theta)$ with $\theta>0$ where $-\infty<\theta<\infty$.

A test procedure is proposed based on the statistic which is given by

$$
\begin{aligned}
U_{m n} & =U_{2}-U_{1} \\
& =\frac{1}{\left(\begin{array}{c}
n \\
k+3
\end{array}\right)} \sum_{C} h\left(Y_{i_{1}}, Y_{i_{2}}, \ldots, Y_{i_{(k+3)}}\right)-\frac{1}{\left(\begin{array}{c}
m \\
k+3
\end{array}\right)} \sum_{D} h\left(X_{i_{1}}, X_{i_{2}}, \ldots, X_{i_{(k+3)}}\right)
\end{aligned}
$$

where $h\left(X_{1}, X_{2}, \ldots, X_{(k+3)}\right)=\frac{1}{\left(\begin{array}{c}k+3 \\ 3\end{array}\right)} \sum_{A} I\left(\operatorname{MIN}\left(X_{j 1}, X_{j 2}, X_{j 3}\right)>-\operatorname{MAX}\left(X_{j 4}, \ldots, X_{j(k+3)}\right)\right)$ and $C$ is the set of $\left(\begin{array}{c}n \\ k+3\end{array}\right)$ combination of integers $\{1,2, \ldots, n\}, D$ is $\left(\begin{array}{c}m \\ k+3\end{array}\right)$ combinations of integers $\{1,2, \ldots, m\}$ and $A$ is the set of $\left(\begin{array}{c}k+3 \\ 3\end{array}\right)$ combinations of integers $\left\{j_{1}, j_{2}, \ldots, j_{(k+3)}\right\}$.

The test criterion is to reject $H_{0}$ in favour of $H_{1}$ for large values of $U_{m, n}$. That is, reject $H_{0}$ if $U_{m, n}>c$. If the alternative is two sided, that is, $H_{1}: \theta \neq 0$, then reject $H_{0}$ when $U_{m, n}$ is too small or $U_{m, n}$ is too large.

\section{Distributional Properties of $U_{m, n}$}

The mean of $U_{m, n}$ is given by

$$
\begin{aligned}
\gamma(F, G) & =E\left(U_{m, n}\right) \\
& =\gamma(G)-\gamma(F) \\
& =0, \quad \text { under } H_{0}
\end{aligned}
$$

The following theorem gives the asymptotic distribution of $U_{m, n}$.

Theorem 1: Under $H_{0}$, the limiting distribution of $\sqrt{N}\left(U_{m, n}-\gamma(F, G)\right), N=m+n$, as $n \rightarrow \infty$ is normal with mean zero and variance $\sigma^{2}=\frac{\sigma_{0}^{2}}{\lambda(1-\lambda)}$, where $\lambda=\lim _{N \rightarrow \infty} \frac{m}{N}$.

Proof: The proof of the theorem follows from Lehmann [5] by noting that $U_{m, n}$ is a two sample $U$ statistics and the asymptotic variance of $\sqrt{N}\left(U_{m, n}-\gamma(F, G)\right)$ is $\sigma^{2}=\frac{\sigma_{0}^{2}}{\lambda(1-\lambda)}$, where $\lambda=\lim _{N \rightarrow \infty} \frac{m}{N}$ ? and under $H_{0}$, 


$$
\begin{aligned}
\sigma_{0}^{2} & =(k+3)^{2} \xi_{10} \\
& =\operatorname{Cov}\left(h\left(X_{1}, X_{2}, \ldots, X_{k+3} ; Y_{1}, Y_{2}, \ldots, Y_{k+3}\right), h\left(X_{1}, X_{k+4}, \ldots, X_{2 k+5} ; Y_{1}, Y_{k+4}, \ldots, Y_{2 k+5}\right)\right) \\
& =(k+3)^{2} \int_{-\infty}^{\infty}\left(\left(\begin{array}{c}
k+2 \\
2
\end{array}\right) A+\left(\begin{array}{c}
k+2 \\
3
\end{array}\right) B\right)^{2} d F(x)-\gamma^{2}(F, G)
\end{aligned}
$$

with $A=\frac{k}{k+2}\left[1-\bar{F}^{k+2}(x)\right]$ and $B=\frac{k-1}{k+2}+\frac{3}{k+2} F^{k+2}(x)$.

In the following table 1 , we tabulate asymptotic variance of $\sqrt{N}\left(U_{m, n}\right)$ under $H_{0}$ for different values of $k$.

Table 1. Asymptotic null variance of $\sqrt{N}\left(U_{m, n}\right)$

\begin{tabular}{cccccccc}
\hline$k$ & 2 & 3 & 4 & 5 & 6 & 7 & 8 \\
\hline Variance & 0.492857 & 0.586753 & 0.614718 & 0.612066 & 0.595542 & 0.573087 & 0.548568 \\
\hline
\end{tabular}

\section{Asymptotic Relative Efficiency (ARE)}

In this section we first obtain the Pitman asymptotic relative efficiency of $U_{m, n}$, with respect to the classical t-test. For this we compute the efficacy of $U_{m, n}$, given by

$$
\begin{aligned}
\operatorname{eff}\left(U_{m, n}\right) & =\left[\lim _{n \rightarrow \infty} \frac{\gamma^{\prime}(F, G)}{\sqrt{N} \operatorname{Var}_{o}\left(U_{m, n}\right)}\right]^{2} \\
& =\frac{\left[6 k \int_{-\infty}^{\infty} F^{k+1}(y) f^{2}(y) d y\right]^{2}}{(k+3)^{2} \xi_{0}}
\end{aligned}
$$

The asymptotic relative efficiencies of the proposed test $U_{m, n}$ with respect to Wilcoxon's test $(\mathrm{W})$, Fisher and Randles (RS) test, Mood's Median test (M-test), Gastwirth H and L tests, Normal Scores(NS) test, Shetty and Govindarajulu test (SG), Shetty and Bhat $(\mathrm{T}(1,3), \mathrm{T}(1,5), \mathrm{T}(2,3), \mathrm{T}(2,5))$ tests are evaluated. Table 2 to Table 12 give the $\operatorname{AREs} \operatorname{eff}\left(U_{m, n}\right)$ for various values of $k$.

Table 2. ARE's of $U_{m, n}$ relative to Wilcoxon's $(W)$ test

\begin{tabular}{cccccc}
\hline$A R E\left(U_{m, n}, W\right)$ & $k=2$ & $k=3$ & $k=4$ & $k=5$ & $k=6$ \\
\hline Laplace & 1.5808 & 1.463733 & 1.334533 & 1.211067 & 1.100467 \\
Logistic & 0.973919 & 0.939084 & 0.896407 & 0.850903 & 0.805946 \\
Uniform & 1.5217 & 1.8406 & 2.169 & 2.5007 & 2.8335 \\
Triangular & 2.161998 & 2.214985 & 2.248509 & 2.269659 & 2.283271 \\
\hline
\end{tabular}

Table 3. ARE's of $U_{m, n}$ relative to Fisher and Randles $(R S)$ test

\begin{tabular}{cccccc}
\hline$A R E\left(U_{m, n}, R S\right)$ & $k=2$ & $k=3$ & $k=4$ & $k=5$ & $k=6$ \\
\hline Laplace & 1.975723 & 1.829373 & 1.667879 & 1.513622 & 1.375313 \\
Logistic & 1.217881 & 1.174381 & 1.120994 & 1.064024 & 1.007795 \\
Uniform & 1.902801 & 2.301607 & 2.712255 & 3.127023 & 3.543165 \\
Triangular & 2.702311 & 2.767437 & 2.810509 & 2.836927 & 2.853926 \\
\hline
\end{tabular}


Table 4. $A R E$ 's of $U_{m, n}$ relative to Mood's Median $(M-t e s t)$ test

\begin{tabular}{cccccc}
\hline$A R E\left(U_{m, n}, M\right)$ & $k=2$ & $k=3$ & $k=4$ & $k=5$ & $k=6$ \\
\hline Laplace & 1.184962 & 1.097187 & 1.000329 & 0.907812 & 0.82486 \\
Logistic & 1.299086 & 1.252685 & 1.195739 & 1.13497 & 1.074992 \\
Uniform & 4.571866 & 5.530078 & 6.516745 & 7.513309 & 8.513173 \\
Triangular & 2.882528 & 2.951997 & 2.997942 & 3.026121 & 3.044254 \\
\hline
\end{tabular}

Table 5. $A R E$ 's of $U_{m, n}$ relative to Gastwirth $(H)$ test

\begin{tabular}{cccccc}
\hline$A R E\left(U_{m, n}, H\right)$ & $k=2$ & $k=3$ & $k=4$ & $k=5$ & $k=6$ \\
\hline Laplace & 1.404028 & 1.300026 & 1.185262 & 1.075641 & 0.977353 \\
Logistic & 1.031424 & 0.994584 & 0.949371 & 0.901123 & 0.853502 \\
Uniform & 3.044437 & 3.682517 & 4.339545 & 5.003164 & 5.668981 \\
Triangular & 2.586558 & 2.648894 & 2.690122 & 2.715408 & 2.731679 \\
\hline
\end{tabular}

Table 6. $A R E$ 's of $U_{m, n}$ relative to Gastwirth $(L)$ test

\begin{tabular}{cccccc}
\hline \multirow{2}{*}{$A R E\left(U_{m, n}, L\right)$} & $k=2$ & $k=3$ & $k=4$ & $k=5$ & $k=6$ \\
\hline Laplace & 3.160685 & 2.92656 & 2.668208 & 2.421434 & 2.200173 \\
Logistic & 1.251979 & 1.207261 & 1.15238 & 1.093814 & 1.036011 \\
Uniform & 0.761165 & 0.920696 & 1.084965 & 1.250882 & 1.417348 \\
Triangular & 2.161896 & 2.213998 & 2.248456 & 2.269591 & 2.283191 \\
\hline
\end{tabular}

Table 7. ARE's of $U_{m, n}$ relative to Normal Scores $(N S)$ test

\begin{tabular}{cccccc}
\hline$A R E\left(U_{m, n}, N S\right)$ & $k=2$ & $k=3$ & $k=4$ & $k=5$ & $k=6$ \\
\hline Laplace & 1.86619 & 1.727954 & 1.575413 & 1.429707 & 1.299067 \\
Logistic & 1.016346 & 0.980044 & 0.935492 & 0.887949 & 0.841025 \\
Uniform & $\infty$ & $\infty$ & $\infty$ & $\infty$ & $\infty$ \\
Triangular & 1.704061 & 1.745128 & 1.77229 & 1.788949 & 1.799668 \\
\hline
\end{tabular}

Table 8. $A R E$ 's of $U_{m, n}$ relative to Shetty and Govindarajulu $(S G)$ test

\begin{tabular}{cccccc}
\hline$A R E\left(U_{m, n}, S G\right)$ & $k=2$ & $k=3$ & $k=4$ & $k=5$ & $k=6$ \\
\hline Laplace & 1.422456 & 1.317089 & 1.200818 & 1.089758 & 0.99018 \\
Logistic & 1.002352 & 0.96655 & 0.922611 & 0.875723 & 0.829445 \\
Uniform & 2.588803 & 3.131386 & 3.690083 & 4.254384 & 4.820553 \\
Triangular & 2.448455 & 2.507463 & 2.546489 & 2.570425 & 2.585827 \\
\hline
\end{tabular}

Table 9. ARE's of $U_{m, n}$ relative to Shetty and Bhat $(T(2,3))$ test

\begin{tabular}{cccccc}
\hline ARE $\left(U_{m, n}, T(2,3)\right)$ & $k=2$ & $k=3$ & $k=4$ & $k=5$ & $k=6$ \\
\hline Laplace & 1.526163 & 1.413143 & 1.288408 & 1.169209 & 1.062432 \\
Logistic & 1.020238 & 0.983747 & 0.939039 & 0.891371 & 0.844276 \\
Uniform & 2.29552 & 2.776588 & 3.271987 & 3.772364 & 4.2744 \\
Triangular & 2.43578 & 2.495476 & 2.533246 & 2.557074 & 2.57241 \\
\hline
\end{tabular}


Table 10. $A R E$ 's of $U_{m, n}$ relative to Shetty and Bhat $(T(1,3))$ test

\begin{tabular}{cccccc}
\hline $\operatorname{ARE}\left(U_{m, n}, T(1,3)\right)$ & $k=2$ & $k=3$ & $k=4$ & $k=5$ & $k=6$ \\
\hline Laplace & 1.4742 & 1.365 & 1.2445 & 1.1294 & 1.0262 \\
Logistic & 0.9855 & 0.9503 & 0.9071 & 0.861 & 0.8155 \\
Uniform & 2.2172 & 2.6819 & 3.1604 & 3.6437 & 4.1286 \\
Triangular & 2.3527 & 2.4094 & 2.4469 & 2.4699 & 2.4847 \\
\hline
\end{tabular}

Table 11. ARE's of $U_{m, n}$ relative to Shetty and Bhat $(T(1,5))$ ? test

\begin{tabular}{cccccc}
\hline ARE $\left(U_{m, n}, T(1,5)\right)$ & $k=2$ & $k=3$ & $k=4$ & $k=5$ & $k=6$ \\
\hline Laplace & 1.422478 & 1.317136 & 1.200876 & 1.089775 & 0.990252 \\
Logistic & 1.001975 & 0.966136 & 0.92223 & 0.875414 & 0.829162 \\
Uniform & 2.727061 & 3.298566 & 3.887097 & 4.481541 & 5.077957 \\
Triangular & 2.595436 & 2.659045 & 2.699291 & 2.724681 & 2.741022 \\
\hline
\end{tabular}

Table 12. $A R E$ 's of $U_{m, n}$ relative to Shetty and Bhat $(T(2,5))$ test

\begin{tabular}{cccccc}
\hline ARE $\left(U_{m, n}, T(2,5)\right)$ & $k=2$ & $k=3$ & $k=4$ & $k=5$ & $k=6$ \\
\hline Laplace & 1.454813 & 1.347077 & 1.228174 & 1.114547 & 1.012762 \\
Logistic & 1.02507 & 0.988406 & 0.943487 & 0.895593 & 0.848275 \\
Uniform & 2.647816 & 3.202714 & 3.774143 & 4.351314 & 4.930398 \\
Triangular & 2.504051 & 2.565421 & 2.60425 & 2.628746 & 2.644512 \\
\hline
\end{tabular}

\section{Some Remarks and Conclusions}

1. A class of test statistics for two-sample location problem is considered in the paper assuming that the underlying distribution of the sample drawn is symmetric.

2. The asymptotic variance of the few members, $U_{m, n}$ (for $k=2,3,4,5,6$ ) of the class of test statistics are computed as a ready reference.

3. The performance of the members of the proposed class is evaluated in terms of asymptotic relative efficiencies (AREs).

4. From table 2 to table 12 , it is observed that the performance of the proposed test is better than the tests existing in the literature for this problem if the distributions of the samples drawn are Laplace, Logistic, Triangular, or Uniform.

5. For heavy tailed distributions such as Laplace and logistic distributions, the performance in terms of ARE decreases with $k$ (that is with subsample size).

6. For light tailed distributions such as Triangular and Uniform the performance in terms of ARE increases with $k$ (that is with subsample size).

\section{References}

1. Ahmad, I.A.(1996). A class of Mann-Whitney-Wilcoxon type statistics. The American Statistician, Vol.50, No.4, 324-327.

2. Gastwirth, J. L.(1965). Percentile modifications of two sample rank tests, Journal of Amer. Statist. Assoc, 0, 1127-1141.

3. Hajek,J. and Sidak, Z.(1967).Theory of rank tests, Academic Press, New York.

4. Hogg,R.V., Fisher,D.M., and Randles,R.H.(1975). A two-sample adaptive distribution-free test. Journal of Amer. Statist. Assoc.70, 656-61. 
5. Lehmann,E.L.(1951).Consistency and unbiasedness of certain nonparametric tests, Ann. Math. Statist., 22,165179.

6. Lehmann, E.L.and D' Abrera, H.J.M. (2006). Nonparametrics: Statistical Methods based on Ranks, SringerVerlag, New York.

7. Mann,H.B. and Whitney,D.R.(1947).On a test of whether one of two random variables is stochastically larger than other, Ann.Math.Statist.,18,50-60.

8. Mathisen, H.C.(1943). A method of testing the hypothesis that two-samples are from the same population, Ann. Math. Statist.14, 188-194.

9. Randles, R. H. and Wolfe, D.A. (1979). Introduction to the Theory of Nonparametric Statistics, John Wiley and Sons, New York.

10. Shetty, I.D. and Z. Govindarajulu(1988). A two-sample test for location, Comm. Statist-Theory and Meth.17, 2389-2401.

11. Shetty, I.D. and Bhat, S.V.(1994). A note on the generalization of Mathisen's median test, Statistics and Probability letters, 19,199-204. 